\title{
Principales variantes anatómicas en bronquios y arterias segmentarias en pulmones humanos
}

\section{Main anatomical variants in bronchi and segmental arteries in human lungs}

\author{
Sirian Saladrigas Sarduy ${ }^{1}$, Armando Méndez Pimentel ${ }^{1}$, Iris Bacallao Cabrera ${ }^{1}$ y Enia Lorenzo Pérez ${ }^{1}$ \\ ${ }^{1}$ Universidad de Ciencias Médicas, Cuba \\ *sirian.cmw@infomed.sld.cu
}

DOI: https://doi.org/10.26871/killkana_salud.v4i2.637

\begin{abstract}
Resumen
Contexto: El cáncer de pulmón es la enfermedad causante de la mayor mortalidad por neoplasias malignas, tanto a escala mundial como en Cuba, por lo que el conocimiento de las complejas variaciones anatómicas y un acertado conocimiento de la anatomía individual se ha convertido en algo cada más importante para los cirujanos torácicos generales al ejecutar una cirugía precisa y segura. Objetivo: Describir las principales variantes anatómicas en bronquios y arterias segmentarias en pulmones humanos. Metodología: Se realizó un estudio descriptivo y transversal en 50 bloques de pulmones humanos obtenidos mediante necropsia del departamento de Medicina Legal del Hospital Docente Clínico Quirúrgico Amalia Simoni de Camagüey. Resultados: se observaron las variantes en los bronquios segmentarios según el número, predominaron los bronquios supernumerarios B1, B2 y B3 en ambos pulmones. La mayor parte de las piezas presentó arterias segmentarias supernumerarias en ambos pulmones A1, A2, A3 y A6. Las variantes en el origen tanto en bronquios como en arterias fueron raras. Conclusiones: Las variantes de número predominaron respecto a las de origen, se observaron con mayor frecuencia en los lóbulos superiores de ambos pulmones y predominó la duplicidad.
\end{abstract}

Palabras clave: variantes anatómicas, bronquios segmentarios, arterias segmentarias.

\begin{abstract}
Context: Lung cancer causes the highest mortality from all malignant neoplasms, both worldwide and in Cuba. Therefore, the knowledge of complex anatomical variations and accurate understanding of individual anatomy has become increasingly significant for thoracic surgeons when performing precise and safe surgery. Objective: To Describe the main anatomical variants in bronchial and segmental arteries, in human lungs. Methodology: A descriptive and cross-sectional study was carried out in 50 human lungs blocks, obtained by necropsy, from the Legal Medicine Department, of the Amalia Simoni Clinical and Surgical Teaching Hospital in Camagüey. Results: Observing the variants in the segmental bronchi according to number, the supernumerary bronchi B1, B2 and B3 prevailed in both lungs. Most parts presented supernumerary segmental arteries in both lungs A1, A2, A3 and A6. Variations in the origin, both in bronchi and arteries, were unusual. Conclusions: The number variants prevailed over those of origin, they were most frequently observed in the upper lobes of both lungs and duplication predominated.
\end{abstract}

Keywords: anatomical variants, segmental bronchi, segmental arteries.

\section{Introducción}

El traumatismo de tórax es una entidad donde el $15 \%$ de los pacientes requieren toracotomía y el $25 \%$ sufre lesión vascular importante y necesita por parte del cirujano un conocimiento anatómico adecuado, el cual es imprescindible para el tratamiento quirúrgico. ${ }^{1}$ Existen múltiples reportes que revelan la existencia de variaciones anatómicas en este órgano de manera aislada. ${ }^{2-4}$ Hoy día la TAC permite evaluar la localización exacta de las lesiones y constituye sin duda el examen de elección para el estudio anatómico de ellas.
Con frecuencia se ignoran las variaciones o se alteran inadvertidamente al tratar de ajustarse a la norma. Por lo tanto, hay que esperar variaciones anatómicas al disecar o inspeccionar muestras. ${ }^{4,5} \mathrm{El}$ estudio de los pulmones por tomografía axial computarizada se ha extendido ampliamente y las pequeñas lesiones pulmonares son detectadas a través de este método, en particular la incidencia del carcinoma de células pulmonares cada vez más pequeñas y las no pequeñas que pueden tener un comportamiento biológico más indolente tal como el carcinoma broncoalveolar se ha incrementado cada vez más. ${ }^{6}$ 
El cáncer de pulmón es la enfermedad causante de la mayor mortalidad por neoplasias malignas, tanto a escala mundial como en Cuba. En el país se producen alrededor de 18000 muertes cada año lo que representa el $21.4 \%$ de todos los fallecimientos. ${ }^{7-9}$ Por lo que el conocimiento completo de las complejas variaciones anatómicas y un acertado conocimiento de la anatomía individual se ha convertido en algo cada más importante para los cirujanos torácicos generales al ejecutar una cirugía precisa y segura. ${ }^{6}$

\section{Metodología}

Se realizó un estudio descriptivo y transversal con el objetivo de describir el comportamiento de las principales variantes anatómicas encontradas en la disección de los segmentos pulmonares en 50 bloques de pulmones humanos en la obtenidos mediante necropsia del departamento de Medicina Legal del Hospital Docente Clínico Quirúrgico Amalia Simoni de Camagüey en el período de noviembre del 2016 a mayo del 2018.

Como criterios de exclusión se consideraron Fallecidos cuyas causas de muerte fueron de etiología cardiorrespiratorias. Fallecidos operados de pulmón. Fallecidos cuyas piezas fueron dañadas por el método de extracción. Las piezas fueron lavadas y perfundidas con agua común y fijada en una solución de formalina al $5 \%$ durante un período de más de tres meses.

Se realizó la disección macroscópica de los pulmones sin separar los derechos de izquierdos. Los datos se procesaron mediante el paquete estadístico SPSS para Windows versión 21.0, y se aplicó la estadística descriptiva. Los resultados se presentaron en tablas.

\section{Resultados}

En la Tabla 1 se observan las variantes en los bronquios segmentarios según el número, predominaron los bronquios supernumerarios en ambos pulmones especialmente, en el izquierdo donde el $100 \%$ de las variaciones fueron de esta variedad, el pulmón izquierdo mostró 3 piezas con bronquios por defecto. Dentro de los supernumerarios se observó bronquio apical (B1) doble, anterior (B3) doble y posterior (B2) doble.

Tabla 1: Distribución de las variantes de número de los bronquios segmentarios en el pulmón derecho

\begin{tabular}{lccc}
\hline Variantes de numero & Pulmón derecho & Pulmón izquierdo \\
& & & \\
\hline Supernumerarios & 14 & 16 & 100 \\
Por defecto & 3 & $\overline{16}$ & $\overline{100}$ \\
Total & 17 & & \\
\hline
\end{tabular}

Fuente: Pulmones humanos disecados

En cuanto al comportamiento de las variantes de número de las arterias segmentarias según pulmón, podemos observar en la Tabla 2 que la mayor parte de las piezas presentó arterias segmentarias supernumerarias con un $78,5 \%$ en los pulmones derechos y el $100 \%$ en los izquierdos. Las arterias segmentarias supernumerarias en ambos pulmones predominaron en los lóbulos superiores.

Tabla 2: Distribución de las variantes de número de las arterias segmentarias según pulmón

\begin{tabular}{lcccc}
\hline Variantes de numero & Pulmón derecho & Pulmón izquierdo \\
& & & & \\
\hline Supernumerarias & 18 & 78,5 & 32 & 100 \\
Por defecto & 5 & 21,5 & $-\overline{1}$ & - \\
Total & 23 & 100 & 32 & 100 \\
\hline
\end{tabular}

Fuente: Pulmones humanos disecados

Las principales variantes observadas fueron segmentaria apical (A1) doble para el lóbulo superior, la arteria segmentaria posterior (A2) doble y triple, piezas anatómicas con segmentaria anterior (A3) doble, (A3) triple y apical inferior (A6) doble y triple. Se constataron además arterias lingulares dobles y triples. En cuanto a las variantes por defecto se constató la arteria segmentaria posterior (A2) ausente solamente en pulmones derechos.

Como se observa en la Tabla 3 la distribución de las variantes de origen de bronquios segmentarios es poco frecuente, solamente se presentó un espécimen con esta variante y correspondió al pulmón derecho, en este caso el bronquio posterior (B2) se originó del bronquio lobar superior correspondiente, pero a un nivel más alto que lo habitual.

Tabla 3: Distribución de las variantes de origen de los bronquios segmentarios en el pulmón derecho

\begin{tabular}{lcc}
\hline Variantes de origen de bronquios segmentarios & No & $\%$ \\
\hline Origen del bronquio correspondiente & 1 & 100 \\
Origen del bronquio no correspondiente & - & - \\
Origen de otro bronquio segmentario & - & - \\
Total & 1 & 100 \\
\hline
\end{tabular}

Fuente: Pulmones humanos disecados

Tal como se muestra en la Tabla 4 las variantes en el origen de las arterias segmentarias solo se observaron en el pulmón derecho, con un predominio del tronco no correspondiente en posición no habitual con un total de 3 piezas, lo que representó un $50 \%$.Este tipo de variante es ampliamente observada por los estudiosos del tema, los cuales la describen en ambos pulmones, no solamente el pulmón derecho como constatamos en este estudio.

Tabla 4: Distribución de las variantes anatómicas del sistema arterial segmentario del pulmón derecho según origen

\begin{tabular}{lcc}
\hline Variantes de origen de bronquios segmentarios & No & $\%$ \\
\hline Origen directamente de la arteria pulmonar & 1 & 16.67 \\
Origen del tronco no correspondiente & 3 & 50 \\
Origen de otro ramo segmentario & 2 & 33.33 \\
Total & 6 & 100 \\
\hline
\end{tabular}

Fuente: Pulmones humanos disecados 
Las variantes de origen de las arterias segmentarias se comportaron de la siguiente manera: en tres casos se presentó la variante, origen del tronco no correspondiente de la siguiente forma: una de ellas resultó ser un tronco trifurcado distribuido, según se expresa a continuación: la arteria segmentaria posterior (A2), luego la arteria segmentaria apical (A1) y la arteria segmentaria anterior (A3).

En dos piezas se halló a la arteria segmentaria anterior (A3) con nacimiento en el tronco inferior y no del tronco correspondiente, superior. Por otra parte en dos de los casos las arterias segmentarias posteriores (A2) se originaron de la arteria segmentaria apical (A6).En uno de los especímenes las arterias segmentarias se iniciaron directamente de la arteria pulmonar y no del tronco superior o anterior. Al realizar el análisis del sistema arterial en el pulmón izquierdo respecto a esta variable no se presentaron variantes.

\section{Discusión}

Sin lugar a duda, tal como se muestra en esta investigación, son las variaciones en el número de bronquios segmentarios las que con más frecuencia se presentan, ya sea por exceso, cuando es un número mayor de ramas que las habitualmente descritas o por defecto cuando el número es menor. ${ }^{10-12}$ Algunos investigadores se limitan a la descripción de lóbulos y segmentos supernumerarios por su importancia en cuanto a su localización y frecuencia. En un estudio realizado en 263 casos se observaron 214 con variaciones en los bronquios segmentarios del lóbulo superior derecho. ${ }^{13}$ Otro autor coincide con lo anteriormente expuesto pues en una investigación realizada encontró que en el 7,2\% de los casos existían variaciones de B1 y B2 en este lóbulo. ${ }^{14}$

Las arterias supernumerarias son ampliamente citadas en la bibliografía; estudios realizados por Toshiteru $\mathrm{N}^{13}$ describen esta variante en un análisis sobre el patrón broncovascular del lóbulo superior usando angiografía y broncografía en 225 casos, en el $85.6 \%$ la A1 es doble y en el $13.3 \%$ A1 emerge sola del tronco superior. Este autor al estudiar la arteria segmentaria anterior (A3) del lóbulo superior derecho clasifica 5 patrones de distribución de esta rama: se observa en 180 casos con segmentaria anterior doble saliendo del tronco superior y fue la clasificación que más se halla, lo cual coincide con este estudio. Este autor observa además en 5 casos $(1.9 \%)$ A3 rama de la arteria ascendente y A3 rama del tronco inferior.

Otras investigaciones también muestran estos resultados .Milloy ${ }^{15}$ plantea que en un estudio realizado observó las ramas arteriales ascendentes dobles en el $90.3 \%$ de los casos. Llorca ${ }^{10}$ cita que con frecuencia la arteria segmentaria apical (A1) es decir (A1a) y (A1b) doble; a veces (A1) y arteria segmentaria posterior (A2) salen de un tronco común, o la arteria segmentaria posterior (A2) es doble, lo que es igual a (A2a y A2b); es muy importante señalar que en este último caso para realizar la lobectomía superior no bastará con ligar el tronco anterior o sus ramas sino que habrá que ligar la profunda arteria segmentaria posterior doble (A2b). Dicho autor reconoce también la presencia de la arteria segmentaria anterior (A3) doble representada (A3a) y (A3b), coincidiendo con estudios realizados por Onuki, ${ }^{16}$ quien plantea que la variabilidad de las ramas pueden ser desde cuatro hasta ocho para este lóbulo.

Cory ${ }^{14}$ en una de sus clasificaciones de las variantes del lóbulo superior derecho en 13 casos $(8.5 \%)$ muestra que el segmento posterior recibe dos ramas separadamente. Reporta en el $25 \%$ de los casos una o más arterias ascendentes cursando anteriormente para dirigirse a una porción del segmento anterior del lóbulo superior derecho, esta rama ascendente anterior usualmente se origina próxima a la arteria ascendente posterior y debido a esto es particularmente propensa a dañarse durante la cirugía, a menos que la vena del lóbulo superior se divida primero. Ocasionalmente la rama anterior puede ser la única rama ascendente. Según otros autores en el lóbulo superior izquierdo se pueden observar desde 4 hasta 7 ramas segmentarias. ${ }^{14-16}$

La segmentaria lingular es ampliamente citada por los investigadores en sus disecciones. Sivrikoz $\mathrm{M}^{17}$ en operaciones realizadas ha observado casos con 3 arterias lingulares, saliendo al mismo nivel que la apical del lóbulo inferior izquierdo (A6). Cory ${ }^{14}$ ha observado una arteria segmentaria apical, segmentaria anterior y un vaso único bien definido para la lìngula; en otros casos este autor observa una rama segmentaria anterior con dirección a la lìngula sin rama lingular. En cuanto a las variantes de origen Milloy ${ }^{15}$ durante el chequeo pre-operatorio de un paciente, muestra que las arterias segmentarias lingulares izquierdas y las segmentarias basales anterior, lateral y posterior (A8+A9+A10) formaron un tronco común, lo que no se observó en esta investigación.

Podemos concluir que las principales variantes en los bronquios y arterias segmentarias observadas en ambos pulmones fueron de número, especialmente bronquios y arterias supernumerarias tal como describen otros autores. Estas se observaron con mayor frecuencia en los lóbulos superiores de ambos pulmones y predominó la duplicidad. Las variantes en el origen tanto en bronquios como en arterias fueron raras.

\section{Fuente de Financiamiento}

Este estudio es autofinanciado.

\section{Conflicto de Intereses}

No existen conflictos personales, profesionales, financieroso de otro tipo.

\section{Consentimiento Informado}

Los autores cuentan con el consentimiento informado de los pacientes para la investigación, la publicación del caso y sus imágenes. 


\section{Referencias Bibliográficas}

1. Algieri R, Ottone NF, M, Bernadou M, Brofman C. Análisis del conocimiento anatómico de las estructuras del pedículo pulmonar y sus relaciones por cirujanos en formación mediante listas de chequeo. Revista Argentina de Anatomía. 2014;5(4). Available from: http://www. anatomia-argentina.com.ar/RevArgAnatOnl-2014-5.

2. Meenakshi S, Manjunath KY, Balasubramanyam V. Morphological variations of the lung fissures and lobes. Indian J Chest Dis Allied Sci. 2004;46:179-82. Available from: http://medind.nic.in/iae/t04/i3/iaet04i3p179.pdf.

3. Şentürk A, Argüder E, Babaoğlu E, Hezer $\mathrm{H},{ }^{14}$ CananHasanoğlu H. Imagen del tromboembolismo pulmonar mediante ecografía endobronquial. ArchBronconeumol. 2013;49(6). Available from: http:// www.archbronconeumol.org/es/diagnostico-por-imagendel-tromboembolismo/articulo/S0300289612002694/.

4. Canseco León N, Serra S. R .Multidetector 15. computed angiography: a new era in the evaluation of pulmonary thromboembolism. Arch CardiolMéx. 2011;81(2):137-150. Available from: http://web.a.ebscohost.com/ehost/pdfviewer/pdfviewer? vid=3\&sid=0c68b479-fdbf-44fa- 9 bdf-dda8c863e1e4.

5. Suwatanapongched T, Buangam C, Sukkasem W, Choochuay H, Charee P, Siripornpitak S. Visibility and variability of pleural fissures on normal digital chest radiographs of 1,000 healthy adults. Jpn J Radiol. 2015. Available from: http://download-v2.springer.com/static/pdf/670/art.

6. Moore KL, Persaud TV. Embriología Clínica. El desarrollo del ser humano. 7th ed. España: Editorial Elsevier; 2004.

7. Costa Montané DM, Prado Lage Y, Lozano Salazar JL, Plasencia Asorey C, Riesgo Cosme YC. Principales aspectos clínicoepidemiológicos del cáncer de pulmón. MEDISAN. 2011;15(8). Available from: http://scieloprueba.sld.cu/scielo.php?script=sci_ arttext\&pid=S1029-30192011000800008\&lng=es.

8. Hidalgo Rodríguez MT, Rojas Alonso JL, Paneque Acosta CA, Ferrer Ballagas S, Tejeda Alvares I. Presentación atípica de neoplasia de pulmón. CCM. 2014;18(3). Available from: http://scieloprueba.sld.cu/scielo.php?script=sci_ arttext\&pid=S1560-43812014000300019\&lng=es.

9. Ministerio de salud pública. Anuario estadístico de salud. La Habana; 2014. Available from: http://files.sld.cu/dne/ files/2013/04/anuario_2012.pdf.

10. Llorca O. Anatomía humana. 5th ed. Barcelona: Editorial Científico Médica; 1980.

11. Gray. Anatomía de Gray. España: Editorial Hartcourt; 2009.
12. Karangelis D, Tagarakis G, Chlapoutakis S, Papadopoulos D, Roubelakis A, Tsilimingas Na. How many lobes do you see? Journal Of Cardiothoracic Surgery. 2011;6(1). Available from: http://web.ebscohost.com/ehost/pdfviewer/ pdfviewer?sid=9d4ec91b-b8d0-43f3-8f6c-f094abf6a144. 13. Toshiteru N, Kimihiro S, Yoichi O, Kai O, Seiichi K, et al. An analysis of variations in the bronchovascular pattern of the right upper lobe using three-dimensional CT angiography and bronchography. Gen ThoracCardiovascSurg. 2015;63:354-360. Available from: http://downloadv2.springer.com/static/pdf/846/art.

4. Cory R, Valentine E. Varying patterns of the lobar branches of the pulmonary artery. A study of 524 lungs and lobes seen at operation of 426 patients. Thorax. 1959. Available from: http://web.b.ebscohost.com/ehost/pdfviewer/pdfviewer? vid=3\&sid=03311409-772d-451e-b617-cde016253349.

15. Warren WH, Milloy FJ. Pulmonary Vascular System and Pulmonary Hilum. ThoracSurgClin. 2007:17. Available from: https://www.clinicalkey.es/service/content/pdf/ watermarked/1-s2.0-S1547412706001137.pdf?locale= es_ES.

16. Onuki T, Kanzaki M, Kikkawa T, Isaka T, Sakamoto $\mathrm{K}$, Murasugi Ma. New findings on the threedimensional anatomical relations between the bronchi and pulmonary blood vessels at the pulmonary hilum. Clinical Anatomy. 2015;28(4). Available from: http://web.b.ebscohost.com/ehost/pdfviewer/pdfviewer? vid=3\&sid=d9fdd668-357f-4f7b-9c45-4f91b56b2854.

7. Sivrikoz M, Tulay C. Variations of lobar branches of pulmonary arteries in thoracic surgery patients. SurgRadiolAnat. 2011;33(6). Available from: http://web.ebscohost.com/ehost/pdfviewer/pdfviewer? vid=12\&sid=9d4ec91b-b8d0-43f3-8f6c-f094abf6a144.

Recibido: 10 de enero de 2020

Aceptado: 15 de junio de 2020 